\title{
Hadron-Hadron Interactions in Coulomb Gauge QCD
}

\author{
Sérgio Szpigel, \\ Faculdade de Ciências Biológicas, Exatas e Experimentais, Universidade Presbiteriana Mackenzie \\ Rua da Consolacão 930, 01302-907 São Paulo, Brasil \\ G. Krein, and R. S. Marques de Carvalho \\ Instituto de Física Teórica, UNESP, Rua Pamplona 145, 01405 São Paulo, Brasil
}

Received on 15 August, 2003.

\begin{abstract}
We describe the derivation of an effective Hamiltonian which involves explicit hadron degrees of freedom and consistently combines chiral symmetry and color confinement. We use a method known as Fock-Tani (FT) representation and a quark model formulated in the context of Coulomb gauge QCD. Using this Hamiltonian, we evaluate the dissociation cross section of $J / \psi$ in collision with $\rho$.
\end{abstract}

\section{Introduction}

The experimental observation of $J / \psi$ suppression in ultrarelativistic heavy-ion collisions by NA38 [1] and more recently the anomalous $J / \psi$ suppression in $\mathrm{Pb}+\mathrm{Pb}$ collisions observed by NA50 [2] have attracted much attention as a possible signal for a quark-gluon plasma (QGP) [3].

Such a suppression can be described by phenomenological models either in a QGP [4] or in a hadronic scenario [5]. Several theoretical studies have been described [6] and the subject is still controversial. In this way, microscopic approaches that allows one to consistently treat hadron-hadron interactions in terms of the underlying quark-gluon structure would provide a useful tool for the understanding of this issue.

In a previous work [7] we described a field theoretical method known as Fock-Tani (FT) representation used to derive an effective Hamiltonian involving explicit hadron degrees of freedom and its application to study hadron interactions using a nonrelativistic microscopic quark model. In this paper we consider the extension of the method to a microscopic relativistic quark model formulated in the context of Coulomb gauge QCD which consistently combines chiral symmetry and color confinement[8]-[10]. Our aim is to set up an effective calculational scheme to comprehensively investigate hadronic structure and interactions such as charmonium suppression.

\section{Coulomb Gauge QCD}

The canonical QCD Hamiltonian in the Coulomb gauge, $\nabla \cdot \mathbf{A}=0$, can be written as [11]-[13]:

$$
\begin{aligned}
H & =\int d \mathbf{x} \psi^{\dagger}\left(-i \alpha \cdot \nabla+m_{q} \beta\right) \psi-g \int d \mathbf{x} \psi^{\dagger} \alpha \cdot \mathbf{A} \psi \\
& +\frac{1}{2} \int d \mathbf{x}\left(\mathcal{J}^{-1} \Pi \cdot \mathcal{J} \Pi+\mathbf{B} \cdot \mathbf{B}\right) \\
& +\frac{1}{2} \int d \mathbf{x} d \mathbf{y} \mathcal{J}^{-1} \rho^{a}(x) K_{a b}(\mathbf{x}, \mathbf{y} ; \mathbf{A}) \mathcal{J} \rho^{b}(y)
\end{aligned}
$$

where $m_{q}$ is the current quark mass, $\mathcal{J}=\operatorname{det}(\nabla \cdot D)$ is the Faddeev-Popov determinant and $D^{a b}=\delta^{a b} \nabla+i g f^{a b c} \mathbf{A}^{c}$ is the covariant derivative in the adjoint representation.

The term $K_{a b}$ is the non-Abelian Coulomb kernel

$$
K_{a b}(\mathbf{x}, \mathbf{y} ; \mathbf{A}) \equiv\left\langle\mathbf{x}, a\left|\frac{g}{\nabla \cdot \mathbf{D}}\left(-\nabla^{2}\right) \frac{g}{\nabla \cdot \mathbf{D}}\right| \mathbf{y}, b\right\rangle
$$

where $\rho^{a}$ is the full color charge density given by

$$
\begin{aligned}
\rho^{a}(\mathbf{x}) & =\rho_{g}^{a}(\mathbf{x})+\rho_{q}^{a}(\mathbf{x}) \\
& =f^{a b c} \mathbf{A}^{b}(\mathbf{x}) \cdot \mathbf{\Pi}^{c}(\mathbf{x})+\psi^{\dagger}(\mathbf{x}) \frac{\lambda^{a}}{2} \psi(\mathbf{x})
\end{aligned}
$$

Note that in the Abelian limit, $\mathbf{D} \rightarrow \nabla$, the QED Coulomb interaction is recovered.

$$
K \rightarrow-g^{2}\left\langle\mathbf{x}, a\left|1 / \nabla^{2}\right| \mathbf{y}, b\right\rangle=g^{2} \delta^{a b} / 4 \pi|\mathbf{x}-\mathbf{y}| .
$$

The dynamical degrees of freedom are the transverse gauge fields $\mathbf{A}^{a}$, the transverse conjugate gluon momenta $\boldsymbol{\Pi}^{a}$ and the quark field $\psi$.

The key features of the Coulomb gauge are [14]: a) The elimination of non-dynamical degrees of freedom creates a long-range instantaneous non-Abelian Coulomb interaction, which provides a confinement scenario: infrared divergences make colored states infinitely heavy, removing them from the physical spectrum; color neutral states, on the other hand, remain physical; b) The absence of spurious degrees of freedom yields Fock states with positive normalizations. This is essential to build nonperturbative models for the QCD vacuum and a quasiparticle basis of constituent quarks and gluons.

\section{Quark Model with Chiral Symme- try Breaking}

The starting point of our model is an approximate QCD Hamiltonian in the Coulomb gauge, in which we use an ef- 
fective Coulomb kernel $K_{a b}(\mathbf{x}, \mathbf{y} ; \mathbf{A}) \rightarrow V(\mathbf{x}-\mathbf{y})$ as obtained in Ref. [14]. This is obtained making $\mathcal{J} \rightarrow 1$ and neglecting quarks in Eq. (3), $\rho_{q}^{a}(\mathbf{x})=0$. The derivation is based on a self-consistent method to construct a gluonic quasiparticle basis. The kernel can be interpreted as an effective interaction between two heavy quarks and the results remarkably well with lattice computations [15]. For long distances, the numerical results for $V(\mathbf{x}-\mathbf{y})$ are almost identical to $V(\mathbf{x}-\mathbf{y})=\sigma|\mathbf{x}-\mathbf{y}|$ and is, therefore, infrared singular and needs in general a careful regularization when dealing with numerical simulations. In the present paper, for simplicity of explaining the model and methods employed to construct an effective hadron-hadron interaction, we use a simpler form for $V(\mathbf{x}-\mathbf{y})$ (see below). However, it should be clear that the methods developed here are not dependent on the specific choice of the kernel.

With such a kernel, the general form of the model Hamiltonian in the fermionic sector is:

$$
\hat{H}=\int d \mathbf{x}\left[\hat{H}_{0}(\mathbf{x})+\hat{H}_{I}(\mathbf{x})\right]
$$

where $\hat{H}_{0}$ is the Hamiltonian density of the Dirac field operator $\psi(\mathbf{x})$,

$$
\hat{H}_{0}(\mathbf{x})=\psi^{\dagger}(\mathbf{x})\left(m_{q} \beta-i \alpha . \nabla\right) \psi(\mathbf{x}),
$$

and $\hat{H}_{I}$ is an effective instantaneous interaction term

$\hat{H}_{I}(\mathbf{x})=\frac{1}{2} \int d \mathbf{y} \psi^{\dagger}(\mathbf{x}) \frac{\lambda^{a}}{2} \psi(\mathbf{x}) V(\mathbf{x}-\mathbf{y}) \psi^{\dagger}(\mathbf{y}) \frac{\lambda^{a}}{2} \psi(\mathbf{y})$.

The next step consists in constructing an approximate new vacuum state for the Hamiltonian in the form of a pairing ansatz $[8,10]$. Let's first define a "trivial" vacuum $|0\rangle$ through $b_{f s c}^{0}|0\rangle=d_{f s c}^{0}|0\rangle=0$, where $b^{0}$ and $d^{0}$ are quark annihilation operators, in terms of which the quark field operator is given by

$$
\psi(\mathbf{x})=\int \frac{d \mathbf{p}}{(2 \pi)^{3 / 2}}\left[u_{s}^{0}(\mathbf{p}) b_{s}^{0}(\mathbf{p})+v_{s}^{0}(\mathbf{p}) d_{s}^{0 \dagger}(-\mathbf{p})\right] e^{i \mathbf{p} \cdot \mathbf{x}}
$$

where color and flavor indices have been neglected. Then, a nontrivial vacuum $|\tilde{0}\rangle$ can be defined through a BogoliubovValatin transformation such as $b|\tilde{0}\rangle=d|\tilde{0}\rangle=0$, where the $b$ and $d$ quark annihilation operators are related to the bare operators $b^{0}$ and $d^{0}$ by the BVT. In terms of the dressed quark operators, the quark field operator can be expanded as

$$
\psi(\mathbf{x})=\int \frac{d \mathbf{p}}{(2 \pi)^{3 / 2}}\left[u_{s}(\mathbf{p}) b_{s}(\mathbf{p})+v_{s}(\mathbf{p}) d_{s}^{\dagger}(-\mathbf{p})\right] e^{i \mathbf{p} \cdot \mathbf{x}},
$$

with the quasiparticle spinors $u_{s}, v_{s}$ given in terms of the $u_{s}^{0}$ and $v_{s}^{0}$ spinors as $[8,10]$

$u_{s}(\mathbf{p})=\frac{1}{\sqrt{2}}[\sqrt{1+\sin \varphi(p)}+\sqrt{1-\sin \varphi(p)} \hat{\mathbf{p}} \cdot \vec{\alpha}] u_{s}^{0}$,
$v_{s}(\mathbf{p})=\frac{1}{\sqrt{2}}[\sqrt{1+\sin \varphi(p)}-\sqrt{1-\sin \varphi(p)} \hat{\mathbf{p}} \cdot \vec{\alpha}] v_{s}^{0}$,

where $\varphi(p)$ is sometimes called the chiral angle and is determined by a gap equation (see below).
The normal order of the Hamiltonian relatively to the new vacuum gives:

$$
\hat{H}=H_{0}+\hat{H}_{2}+\hat{H}_{2}^{A}+\hat{H}_{4},
$$

where $H_{0}$ is a constant and gives the energy of the new vacuum, and

$$
\hat{H}_{2}=\int d \mathbf{p} E(\mathbf{p})\left[b_{s}^{\dagger}(\mathbf{p}) b_{s}(\mathbf{p})+d_{s}^{\dagger}(-\mathbf{p}) d_{s}(-\mathbf{p})\right],
$$

where $E(p)$ is the energy of a free quark:

$$
\begin{aligned}
& E(\mathbf{p})=\sin \varphi(p) A(\mathbf{p})+\cos \varphi(p) B(\mathbf{p}) \\
& A(\mathbf{p})=m_{q}+\frac{2}{3} \int d \mathbf{k} V(\mathbf{p}-\mathbf{k}) \sin \varphi(p) \\
& B(\mathbf{p})=p+\frac{2}{3} \int d \mathbf{k} V(\mathbf{p}-\mathbf{k}) \cos \varphi(p) \hat{\mathbf{p}} . \hat{\mathbf{k}},
\end{aligned}
$$

and

$$
\begin{aligned}
\hat{H}_{4}= & \frac{1}{2} \int d \mathbf{p} d \mathbf{k} d \mathbf{q} V(\mathbf{q})\left(\frac{\lambda_{c_{1} c_{2}}^{a} \lambda_{c_{1} c_{2}}^{a}}{4}\right) \\
& \times \sum_{j, l=1}^{4}: \Theta_{c_{1} c_{2}}^{j}(\mathbf{p}, \mathbf{p}+\mathbf{q}) \Theta_{c_{3} c_{4}}^{l}(\mathbf{k}, \mathbf{k}-\mathbf{q})
\end{aligned}
$$

gives 10 different terms that are combinations of the following four vertices (here we have introduced the color indices for clarity):

$$
\begin{aligned}
& \Theta_{c^{\prime} c}^{1}\left(\mathbf{p}, \mathbf{p}^{\prime}\right) \equiv u_{s^{\prime}}^{\dagger}\left(\mathbf{p}^{\prime}\right) u_{s}(\mathbf{p}) b_{s^{\prime} c^{\prime}}^{\dagger}\left(\mathbf{p}^{\prime}\right) b_{s c}(\mathbf{p}), \\
& \Theta_{c^{\prime} c}^{2}\left(\mathbf{p}, \mathbf{p}^{\prime}\right) \equiv-v_{s^{\prime}}^{\dagger}\left(\mathbf{p}^{\prime}\right) v_{s}(\mathbf{p}) d_{s c}^{\dagger}(-\mathbf{p}) d_{s^{\prime} c^{\prime}}\left(-\mathbf{p}^{\prime}\right), \\
& \Theta_{c^{\prime} c}^{3}\left(\mathbf{p}, \mathbf{p}^{\prime}\right) \equiv u_{s^{\prime}}^{\dagger}\left(\mathbf{p}^{\prime}\right) v_{s}(\mathbf{p}) d_{s^{\prime} c^{\prime}}^{\dagger}\left(\mathbf{p}^{\prime}\right) d_{s c}^{\dagger}(-\mathbf{p}) \\
& \Theta_{c^{\prime} c}^{4}\left(\mathbf{p}, \mathbf{p}^{\prime}\right) \equiv v_{s^{\prime}}^{\dagger}\left(\mathbf{p}^{\prime}\right) u_{s}(\mathbf{p}) d_{s^{\prime} c^{\prime}}^{\dagger}\left(-\mathbf{p}^{\prime}\right) b_{s c}(\mathbf{p}) .
\end{aligned}
$$

The term $\hat{H}_{2}^{A}$ is the anomalous, nondiagonal Bogoliubov term. In order to bring the single-quark Hamiltonian into a diagonal form, one has to require $\hat{H}_{2}^{A}=0$, which leads to the gap equation

$$
A(\mathbf{p}) \cos \varphi(p)-B(\mathbf{p}) \sin \varphi(p)=0 .
$$

It is useful to introduce a running quasiparticle quark mass, $M(\mathbf{p})$, through the equations

$$
\cos \varphi(p)=\frac{p}{E(\mathbf{p})}, \quad \sin \varphi(p)=\frac{M(\mathbf{p})}{E(\mathbf{p})}
$$

with $E(\mathbf{p})=\sqrt{\mathbf{p}^{2}+M^{2}(\mathbf{p})}$. One can identify an effective constituent quark mass as $M_{q}=\max [M(\mathbf{p})]$ and extract it from the low momentum behavior of the chiral angle [16].

\section{Effective Hadron-Hadron Hamil- tonian}

Effective hadron-hadron potentials in quark potential models have been obtained within several early approaches such 
as adiabatic methods [17], resonating group [18], variational techniques [19] and the QBD formalism [20]. In this work we use the Fock-Tani (FT) formalism, which was developed independently by Girardeau [21] and Vorob'ev and Khomkin [22] in the context of atomic physics and has recently been extended to hadronic physics [7]. The method shares some similarities with Weinberg's quasi-particle approach [23].

In the following, we present the main features of the Fock-Tani formalism for the derivation of an effective meson-meson interaction. We start by specifying the microscopic Hamiltonian in Fock space $(\mathcal{F})$ :

$$
\begin{aligned}
& H=T(\mu) q_{\mu}^{\dagger} q_{\mu}+T(\nu) \bar{q}_{\nu}^{\dagger} \bar{q}_{\nu}+\frac{1}{2} V_{q q}(\mu \nu ; \sigma \rho) q_{\mu}^{\dagger} q_{\nu}^{\dagger} q_{\rho} q_{\sigma} \\
& +\quad \frac{1}{2} V_{\bar{q} \bar{q}}(\mu \nu ; \sigma \rho) \bar{q}_{\mu}^{\dagger} \bar{q}_{\nu}^{\dagger} \bar{q}_{\rho} \bar{q}_{\sigma}+V_{q \bar{q}}(\mu \nu ; \sigma \rho) q_{\mu}^{\dagger} \bar{q}_{\nu}^{\dagger} \bar{q}_{\rho} q_{\sigma}(18)
\end{aligned}
$$

In Eq.(18), $T$ is the kinetic energy and $V_{q q}, V_{\overline{q q}}$ and $V_{a \bar{q}}$ are respectively the quark-quark, antiquark-antiquark and quark-antiquark interactions. The indices $\mu, \nu, \cdots$ represent spatial, color, spin, and flavor quantum numbers of the quarks and antiquarks and a summation over repeated indices is implied. The quark and antiquark operators obey standard anticommutation relations:

$$
\begin{aligned}
& \left\{\bar{q}_{\mu}, \bar{q}_{\nu}^{\dagger}\right\}=\left\{q_{\mu}, q_{\nu}^{\dagger}\right\}=\delta_{\mu \nu}, \\
& \left\{q_{\mu}, q_{\nu}\right\}=\left\{\bar{q}_{\mu}, \bar{q}_{\nu}\right\}=\left\{\bar{q}_{\mu}, q_{\nu}^{\dagger}\right\}=0 .
\end{aligned}
$$

A generic meson state in $\mathcal{F}$, composed by a quarkantiquark pair, is denoted by $|\alpha\rangle$, where $\alpha$ represents the meson quantum numbers (c.m. momentum, internal energy, spin and flavor). Such a state can be written as:

$$
|\alpha\rangle=M_{\alpha}^{\dagger}|0\rangle \equiv \Phi_{\alpha}^{\mu \nu} q_{\mu}^{\dagger} \bar{q}_{\nu}^{\dagger}|0\rangle,
$$

where $M_{\alpha}^{\dagger}$ is the meson creation operator, $\Phi_{\alpha}^{\mu \nu}$ is the meson wave function and $|0\rangle$ is the vacuum state, defined as $q_{\mu}|0\rangle=\bar{q}_{\nu}|0\rangle=0$. Using the quark anticommutation relations of Eq. (19), and the orthonormalization condition for the $\Phi$ 's, one can show that the meson operators satisfy the following noncanonical commutation relations:

$$
\left[M_{\alpha}, M_{\beta}^{\dagger}\right]=\delta_{\alpha \beta}-\Delta_{\alpha \beta}, \quad\left[M_{\alpha}, M_{\beta}\right]=0,
$$

where $\Delta_{\alpha \beta}=\Phi_{\alpha}^{* \mu \nu} \Phi_{\beta}^{\mu \sigma} \bar{q}_{\sigma}^{\dagger} \bar{q}_{\nu}+\Phi_{\alpha}^{* \mu \nu} \Phi_{\beta}^{\rho \nu} q_{\rho}^{\dagger} q_{\mu}$ is the term that manifests the composite nature of the mesons.

The change to the FT representation is implemented by means of a unitary transformation $U$, such that a single composite meson state $|\alpha\rangle$ is transformed into a single idealmeson state $\left.\mid \alpha)=m_{\alpha}^{\dagger} \mid 0\right) \equiv U^{-1}|\alpha\rangle$, where $m_{\alpha}^{\dagger}$ and $m_{\alpha}$ are the ideal-meson creation and annihilation operators that satisfy canonical commutation relations:

$$
\left[m_{\alpha}, m_{\beta}^{\dagger}\right]=\delta_{\alpha \beta}, \quad\left[m_{\alpha}, m_{\beta}\right]=\left[m_{\alpha}^{\dagger}, m_{\beta}^{\dagger}\right]=0 .
$$

By definition, the $m^{\dagger}$ and $m$ commute with the quark and antiquark operators. In this way, within the FT representation one recovers the possibility of using traditional field theoretic techniques such as Wick's theorem, Feynman diagrams, etc.
The operator $U$ is constructed as a power series in the bound state wavefunctions $\Phi$. Once the operator $U$ is known, one proceeds by transforming the original quarkmodel operators, such as currents and Hamiltonian. This is accomplished by transforming initially the quark and antiquark operators and substituting these into the expressions of quark model operators. The explicit form of $U$ and the derivation of the transformed quark and antiquark operators is discussed in detail in Ref. [7].

The structure of the transformed Hamiltonian is:

$$
H_{\mathrm{FT}}=H_{q}+H_{m}+H_{m q} .
$$

The quark Hamiltonian, $H_{q}$, has an identical structure to the one of the microscopic quark Hamiltonian of Eq. (18), except that the term corresponding to the quark-antiquark interaction is modified such that it does not produce the quark-antiquark bound states. $H_{m q}$ describes quark-meson processes as meson breakup into a quark-antiquark pair, etc. The term involving only ideal meson operators, $H_{m}$, has a component that represents an effective meson-meson interaction:

$$
\begin{aligned}
H_{m m} & =\Phi_{\alpha}^{* \mu \nu} H\left(\mu \nu ; \mu^{\prime} \nu^{\prime}\right) \Phi_{\beta}^{\mu^{\prime} \nu^{\prime}} m_{\alpha}^{\dagger} m_{\alpha} \\
& +\frac{1}{2} \sum_{\alpha \beta \gamma \delta} V_{m m}(\alpha \beta ; \gamma \delta) m_{\alpha}^{\dagger} m_{\beta}^{\dagger} m_{\delta} m_{\gamma}
\end{aligned}
$$

where the effective meson-meson potential $V_{m m}$ is a sum of several different terms involving $H\left(\mu \nu ; \mu^{\prime} \nu^{\prime}\right)$ and the product of four wave-functions corresponding to the initial and final meson states.

Note that the effective meson Hamiltonian is model independent, in the sense that it depends only on the general forms of the microscopic quark Hamiltonian and of the meson states.

\section{Ongoing Calculations}

In order to illustrate the application of the framework through a simple example, we have calculated the scattering cross section for charmonium dissociation by inelastic scattering on $\rho$ mesons, using the effective meson-meson Hamiltonian derived in section 4 and the quark model Hamiltonian with chiral symmetry breaking described in section 3 . Our final aim is to perform the calculation using the potential derived from the gauge sector of the Coulomb gauge QCD Hamiltonian, as in Ref. [14]. However, such an interaction exhibits a strong singularity at $q \rightarrow 0$ that needs to be regulated in the process of performing a numerical integration. We are still in the process of regulating such a numerical singularity (there is no real singularity since the integrands are finite at $q=0$ ). Thus, here we just show the results obtained using a Gaussian interaction given by:

$$
V(\mathbf{q})=\frac{1}{(2 \pi)^{3 / 2}} V_{0}(8 \pi \chi)^{\frac{3}{2}} e^{-2 \chi \mathbf{q}^{2}} .
$$

The $J / \psi$ mesons are composites of a heavy quark and a heavy antiquark pair, denoted by $(Q \bar{Q})$, and the $\rho$ mesons are composites of a light quark and a light antiquark, denoted by $(q \bar{q})$. The final mesons $D, \bar{D}$ are composites of a $(q \bar{Q})$ or a $(Q \bar{q})$ pair and can be either in the fundamental 
$D, \bar{D}\left({ }^{1} 1 S_{0}\right)$ or in the excited $D^{*}, \bar{D}^{*}\left({ }^{3} 1 S_{1}\right)$ states. The explicit form of the creation operator for a composite meson is

$$
\begin{aligned}
M_{C S F}^{\dagger}(\mathbf{p}) & =\sum_{c s f} \mathcal{C}_{C}^{c_{1} c_{2}} \chi_{S}^{s_{1} s_{2}} \mathcal{F}_{F}^{f_{1} f_{2}} \int d \mathbf{k}_{1} d \mathbf{k}_{2} \Phi_{\mathbf{p}}\left(\mathbf{k}_{1}, \mathbf{k}_{2}\right) \\
& \times q_{c_{1} s_{1} f_{1}}^{\dagger}\left(\mathbf{k}_{1}\right) \bar{q}_{c_{2} s_{2} f_{2}}^{\dagger}\left(\mathbf{k}_{2}\right),
\end{aligned}
$$

where $\mathcal{C}_{C}, \chi_{S}$, and $\mathcal{F}_{F}$ are respectively the color, spin and flavor Clebsch-Gordan coefficients. For the spatial meson wave-function we employ a Gaussian ansatz :

$$
\Phi_{\mathbf{p}}^{\mathbf{k}_{1} \mathbf{k}_{2}}=\delta^{(3)}\left(\mathbf{p}-\mathbf{k}_{1}-\mathbf{k}_{2}\right)\left(\frac{b^{2}}{\pi}\right)^{\frac{3}{4}} e^{-b^{2} \overline{\mathbf{k}}^{2} / 2}
$$

where $\overline{\mathbf{k}}=\eta \mathbf{k}_{1}-(1-\eta) \mathbf{k}_{2}$, with $\eta=m_{2} /\left(m_{1}+m_{2}\right)$ and $b$ is the Gaussian parameter related to the r.m.s. radius of the meson by $\left\langle r^{2}\right\rangle=\sqrt{3 / 2} b$.

There are six final state reaction channels for the reaction, allowed by momentum conservation:

$$
J / \psi\left({ }^{3} 1 S_{1}\right)+\rho\left({ }^{3} 1 S_{1}\right) \rightarrow D(1 S)+\bar{D}(1 S) .
$$

The total cross section for the reaction is a function of the center-of-mass energy and is obtained by summing over all possible final channels $\sigma_{t o t}(s)=\sum_{f=1}^{6} \sigma_{f i}(s)$. For numerical evaluations, the parameter values used are:

$$
\begin{aligned}
& m_{Q}=1.67 \mathrm{GeV}, m_{q}=0.33 \mathrm{GeV}, \\
& V_{0}=0.5 \mathrm{GeV}, \chi=1.0 \mathrm{GeV}^{-2}, \\
& b_{Q \bar{Q}}=0.560 \mathrm{GeV}, b_{q \bar{q}}=0.380 \mathrm{GeV}, \\
& b_{Q \bar{q}}=b_{q \bar{Q}}=0.440 \mathrm{GeV} .
\end{aligned}
$$

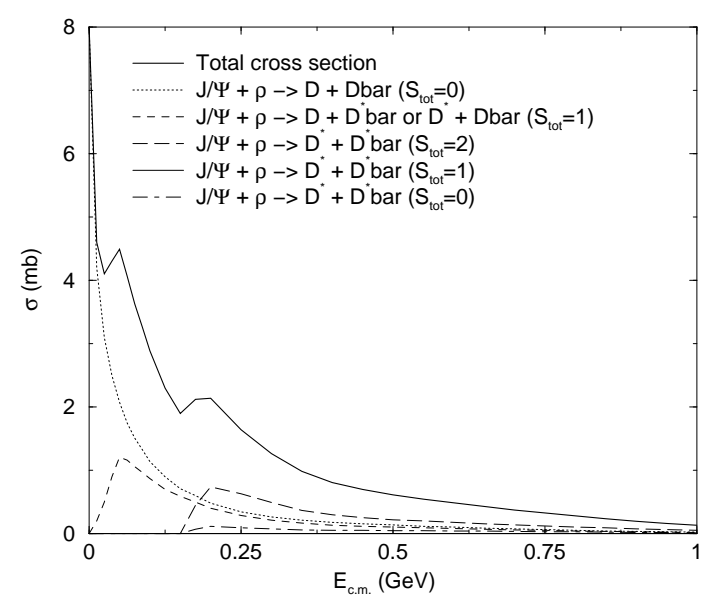

Figure 1. Cross-sections for $J / \psi+\rho$ scattering.

In Fig. 1 we show the cross sections for the reaction as a function of the relative kinetic energy of the $J / \psi$ and the $\rho$ in the center-of-mass system.

\section{References}

[1] M.C. Abreu et. al., Z. Phys. C 38, 117 (1988).

[2] M. Gonin, Nucl. Phys. A610, 404c (1996); M.C. Abreu, Phys. lett. B477, 28 (2000).

[3] T. Matsui and H. Satz, Phys. Lett. B178, 416 (1986).

[4] F. Karsch and H. Satz, Z. Phys. C 51, 209 (1991).

[5] J. Hufner, Y. Kurihara, and H.J. Pirner, Phys. Lett. B 215, 218 (1988).

[6] R. Vogt, S.J. Brodsky, and P. Hoyer, Nucl. Phys. B360, 67 (1991); D. Kharzeev and H. Satz, Phys. Lett. B334, 155 (1994); K. Martins, D. Blaschke, and E. Quack, Phys. Rev. C 51, 2723 (1995); C. Wong, E.S. Swanson, and T. Barnes, Phys. Rev. C 65, 014903 (2002); F.O. Durães, S. H. Lee, F.S. Navarra and M. Nielsen, Phys. Lett. B564, 97 (2003); F.S. Navarra, M. Nielsen, R.S.M. de Carvalho, and G. Krein, Phys. Lett. B529, 87 (2002).

[7] D. Hadjimichef, G. Krein, S. Szpigel, and J.S. da Veiga, Ann. Phys. 268 (1998), 105; Phys. Lett. B 367, 317 (1996).

[8] P.J.A. Bicudo, G. Krein, and J.E.F.T. Ribeiro, Phys. Rev. C 64, 025202 (1991).

[9] P.J.A. Bicudo, G. Krein, J.E.F.T. Ribeiro, and J.E. Villate, Phys. Rev. D 45, 1673 (1992).

[10] P.J.A. Bicudo, L.S. Ferreira, C.M. Plácido, and J.E.F.T. Ribeiro, Phys. Rev. C 56, 670 (1997).

[11] J. Schwinger, Phys. Rev. 127, 324 (1962).

[12] I.B. Khriplovich, Yad. F. 10, 409 (1969).

[13] N.H. Christ and T.D. Lee, Phys. Rev. D 22, 939 (1980).

[14] A. P. Szczepaniak and E. S. Swanson, Phys. Rev. D65, 025012-1 (2002); Phys. Rev. D62, 094027 (2000).

[15] K.J. Juge, J. Kuti, and C.J. Morningstar, Nucl. Phys. B (Proc. Suppl.) 63, 326 (1998).

[16] F.J. Llanes-Estrada, S.R. Cotanch, Nucl.Phys. A697, 303 (2002).

[17] D. A. Liberman, Phys. Rev. D 16, 1542 (1977); C.E. DeTar, Phys. Rev. D 17, 323 (1977); M. Harvey, Nucl. Phys. A352, 326 (1981).

[18] For a review see: K. Shimizu, Rep. Prog. Phys. 52 (1989).

[19] J. Weinstein and N. Isgur, Phys. Rev. Lett. 48, 659 (1982); Phys. Rev. D 27, 588 (1983); 41, 2236 (1990).

[20] T. Barnes and E.S. Swanson, Phys. Rev. D 46, 131 (1992); T. Barnes, S. Capstick, M.D. Kovarik and E.S. Swanson, Phys. Rev. C 48, 539 (1993).

[21] M.D. Girardeau, Phys. Rev. Lett. 27 (1971) 1416.

[22] V.S. Vorob'ev and A.L. Khomkin, Teor. i Mat. Fiz. 8, 109 (1971) (in Russian). English translation: Consultants Bureau, Plenum Publ. Co., New York, 1972.

[23] S. Weinberg, Phys. Rev. 130, 776 (1963); 131, 440 (1963); M. Scadron and S. Weinberg, Phys. Rev. 133, B1589 (1964). 